\title{
Activación alcalina de metacaolín. Efecto de la adición de silicato soluble y de la temperatura de curado
}

\author{
M. MONZÓ', A. FERNÁNDEZ-JIMÉNEZ², M. VICENT' ${ }^{1}$ A. PALOMO ${ }^{2}$, A. BARBA ${ }^{1}$ \\ IInstituto de Tecnología Cerámica. Asociación de Investigación de las Industrias Cerámicas. Universitat Jaume I, Castellón. \\ ${ }^{2}$ Instituto de Ciencias de la Construcción Eduardo Torroja (CSIC), Madrid.
}

\begin{abstract}
El objetivo del presente trabajo es determinar el efecto que la cantidad de sílice soluble presente en la disolución activadora (relación molar $\mathrm{SiO}_{2} / \mathrm{Na}_{2} \mathrm{O}$ entre 0 y 0.69$)$ y la temperatura de curado $\left(85^{\circ} \mathrm{C}, 150^{\circ} \mathrm{C}\right.$ y $200^{\circ} \mathrm{C}$ ) ejercen sobre las propiedades físico-mecánicas y las características mineralógicas y microestructurales de los productos formados por activación alcalina de metacaolín. Para ello se determinaron los valores de resistencia mecánica a compresión de los materiales objeto de estudio y se realizó un estudio mineralógico y microestructural de los mismos por DRX, SEM-EDX, ${ }^{29}$ Si RMN-MAS y porosimetría de mercurio.

Los resultados obtenidos muestran que la resistencia mecánica del material guarda una estrecha relación con la naturaleza, microestructura y composición química de los productos de reacción así como con las condiciones de curado. En todos los casos se genera, como principal producto de reacción, un polímero inorgánico alcalino con propiedades cementantes (gel NA-S-H) responsable, en mayor medida, de las propiedades mecánicas del material. Como productos secundarios se forman algunas zeolitas cuya proporción y tipo (sodalita, zeolita A, faujasita, ...) dependen tanto de la naturaleza del activador como de las condiciones de curado. La presencia de sílice soluble en la disolución activadora conduce a la formación de geles con una mayor relación Si / Al y a la ralentización de la zeolitización, factores, ambos, con un efecto positivo sobre la resistencia mecánica. Con respecto a la temperatura de curado, existe un valor umbral, en función de la composición del material, a partir del cual un incremento en dicha temperatura deja de tener efectos positivos sobre la evolución de la resistencia mecánica.
\end{abstract}

Palabras clave: Metacaolín, activación alcalina, SEM-EDX, ${ }^{29}$ Si RMN-MAS, porosimetría de mercurio.

\section{Alkaline activation of metakaolin. Effect of the soluble silicate addition and curing temperature}

This study has been undertaken to determine the effect that the soluble silica content in the activating solution (molar ratio $\mathrm{SiO}_{2} / \mathrm{Na}_{2} \mathrm{O}$ between 0 and 0.69$)$ and curing temperature $\left(85^{\circ} \mathrm{C}, 150^{\circ} \mathrm{C}\right.$, and $\left.200^{\circ} \mathrm{C}\right)$ have on the physico-mechanical properties and mineralogical and microstructural characteristics of products formed by alkaline activation of metakaolin. The compression strength of the materials obtained was determined and a mineralogical and microstructural study of these materials was conducted by XRD, SEM-EDX, ${ }^{29}$ Si MAS-NMR, and mercury porosimetry.

The results show that the mechanical strength of the material is closely related to the nature, microstructure, and chemical composition of the reaction products, as well as to the curing conditions. In all cases, the main reaction product that forms is an inorganic alkaline polymer with cementing properties (N-A-S-H gel), which is largely responsible for the mechanical properties of the material. Some zeolites form as by-products, whose quantity and type (sodalite, zeolite A, faujasite, etc.) depend on the nature of the activator and the curing conditions. The presence of soluble silica in the activating solution leads to the formation of gels with a larger $\mathrm{Si} / \mathrm{Al}$ ratio and slower zeolitisation, these both being factors with a positive effect on mechanical strength. The curing temperature exhibits a threshold value that depends on the composition of the material, beyond which an increase in curing temperature ceases to positively affect the evolution of the material's mechanical strength.

Keywords: Metakaolin, alkaline activation, SEM-EDX, ${ }^{29}$ Si MAS-NMR, mercury porosimetry.

\section{INTRODUCCIÓN}

La activación alcalina es un proceso químico en el cual un material pulverulento de naturaleza silicoaluminosa, como es el caso del metacaolín, se mezcla con un activador alcalino para generar una pasta con capacidad de fraguar y endurecer en un corto período de tiempo (1-8). El principal producto de reacción en este proceso es un gel de silicoaluminato sódico (gel N-A-S-H), amorfo a la DRX, que presenta estructura tridimensional. Estos materiales, a los que genéricamente se les denomina polímeros inorgánicos alcalinos, y también geopolímeros, geocementos, zeocerámicas, etc., pueden presentar una amplia variedad de propiedades y características (elevada resistencia mecánica inicial, resistencia al fuego, a los ácidos, etc. (8-10)) dependiendo de las condiciones en las que se desarrolla el proceso (tipo de activador, temperatura, 
tiempo, etc.). En definitiva, los polímeros inorgánicos alcalinos constituyen una nueva familia de productos que poseen la capacidad de conjugar cualidades específicas de los cementos con aspectos específicos de la cerámica tradicional, así como atributos que pertenecen a la familia de las zeolitas.

Son numerosas las variables que afectan al proceso de activación alcalina: tipo y composición de los materiales de partida, naturaleza y concentración del activador alcalino, temperatura y tiempo de curado, etc. (1-8). El presente trabajo se centra en el estudio de dos de estas variables, naturaleza y concentración del activador alcalino y temperatura de curado, a fin de determinar la posible relación existente entre ambas.

En lo que respecta al efecto de la naturaleza del activador sobre el proceso químico de formación de polímeros inorgánicos alcalinos, hay que destacar el papel que juegan tanto los cationes alcalinos que se incorporan al sistema como los aniones que acompañan al álcali en la disolución activadora (8-11). Los cationes actúan compensando y equilibrando el balance de carga eléctrica que descompensa la estructura por la sustitución de átomos de $\mathrm{Si}^{4+}$ por átomos de $\mathrm{Al}^{3+}$. Esto ocurre en el proceso de disolución, es decir, cuando la elevada concentración de iones $\mathrm{OH}^{-}$en el medio rompe los enlaces covalentes $\mathrm{Si}-\mathrm{O}-\mathrm{Si}$, Si-O-Al y Al-O-Al presentes en el metacaolín y los iones silicio y aluminio pasan al medio formando grupos Si-OH y Al-OH. Posteriormente estas especies químicas condensan, forman enlaces Si-O-Al y Si-OSi y dan lugar a la precipitación de un gel que se caracteriza por poseer estructura tridimensional. las reacciones. En general, al incrementar la temperatura se reduce el tiempo necesario para alcanzar un valor óptimo de resistencia mecánica $(8,15)$. Así, en estudios previos se observó que para el caso del metacaolín y de mezclas de metacaolín y cenizas volantes, cuando se utilizaba como disolución activadora una mezcla de silicato sódico e hidróxido sódico, los mejores valores de resistencia se obtenían para temperaturas de curado de $150^{\circ} \mathrm{C}(16)$.

Teniendo en cuenta estas consideraciones, el objetivo del presente trabajo es estudiar el efecto de la cantidad de sílice soluble presente en la disolución activadora y el de la temperatura de curado sobre las propiedades mecánicas y la naturaleza y composición de los productos formados en la activación alcalina de metacaolín.

\section{EXPERIMENTAL}

La materia prima utilizada en este trabajo ha sido metacaolín obtenido calcinando, a $750^{\circ} \mathrm{C}$ durante 20 horas $(4,5)$, caolín de CAOBAR, cuyas minas se encuentran en la provincia de Guadalajara. El caolín es una materia prima natural que se obtiene a partir del beneficio de caolín bruto o roca de caolín recién extraída y que contiene caolinita como mineral principal.

La composición química del metacaolín (Tabla I) se determinó por fluorescencia de rayos X (FRX). El equipo utilizado fue un espectrómetro de la firma PHILIPS, modelo

TABLA I. ANÁLISIS QUIIMICO DEL METACAOLÍN.

\begin{tabular}{|c|c|c|c|c|c|c|c|c|c|c|}
\hline \multicolumn{10}{|c|}{ \% óxidos (en peso) } \\
\hline $\mathrm{SiO}_{2}$ & $\mathrm{Al}_{2} \mathrm{O}_{3}$ & $\mathrm{Fe}_{2} \mathrm{O}_{3}$ & $\mathrm{CaO}$ & $\mathrm{MgO}$ & $\mathrm{Na}_{2} \mathrm{O}$ & $\mathrm{K}_{2} \mathrm{O}$ & $\mathrm{TiO}_{2}$ & $\mathrm{MnO}$ & $\mathrm{P}_{2} \mathrm{O}_{5}$ & $\left(^{*}\right) \mathrm{ppc}$ \\
\hline 57.0 & 41.0 & 0.48 & 0.10 & 0.10 & $<0.01$ & 0.50 & 0.24 & $<0.01$ & 0.05 & 0.44 \\
\hline
\end{tabular}

(*) Pérdida por calcinación a $1025^{\circ} \mathrm{C}$

TABLA II. CARACTERIZACIÓN FÍSICA DEL METACAOLÍN.

\begin{tabular}{|c|c|c|c|}
\hline \multicolumn{2}{|c|}{ Análisis granulométrico (luz de malla) } & \multirow{2}{*}{$\rho_{\text {real }}\left(\mathrm{g} / \mathrm{cm}^{3}\right)$} & \multicolumn{2}{c|}{$\mathrm{S}_{\text {esp }} \mathrm{BET}\left(\mathrm{m}^{2} / \mathrm{g}\right)$} \\
\cline { 1 - 3 } $\mathrm{d}<45 \mu \mathrm{m}$ & $\mathrm{d}>45 \mu \mathrm{m}$ & 2.57 \\
\hline $100 \%$ & --- & 7.7 \\
\hline
\end{tabular}

Por su parte, el anión que acompaña al álcali en la disolución activadora ejerce un efecto importante en el desarrollo de las reacciones de activación alcalina y, en consecuencia, en las características mineralógicas y microestructurales de los materiales sintetizados $(6,8,11,12)$. En estudios previos sobre el efecto de la sílice soluble en la activación de cenizas volantes y de metacaolín, se ha demostrado que, en general, la presencia de sílice soluble en la disolución activadora incrementa la resistencia mecánica inicial del material (4-8). No obstante, existe un valor umbral por encima del cual el incremento del contenido de sílice deja de tener los efectos positivos deseados.

Si bien la mayoría de los autores (13-15) coinciden en afirmar que los valores más altos de resistencia se obtienen cuando se forman geles con composiciones $\mathrm{Si} / \mathrm{Al} \approx 2$, no está claro el efecto de la sílice soluble en la microestructura de los productos de reacción y cómo ésta se ve alterada por la temperatura de curado, factor determinante en la cinética de

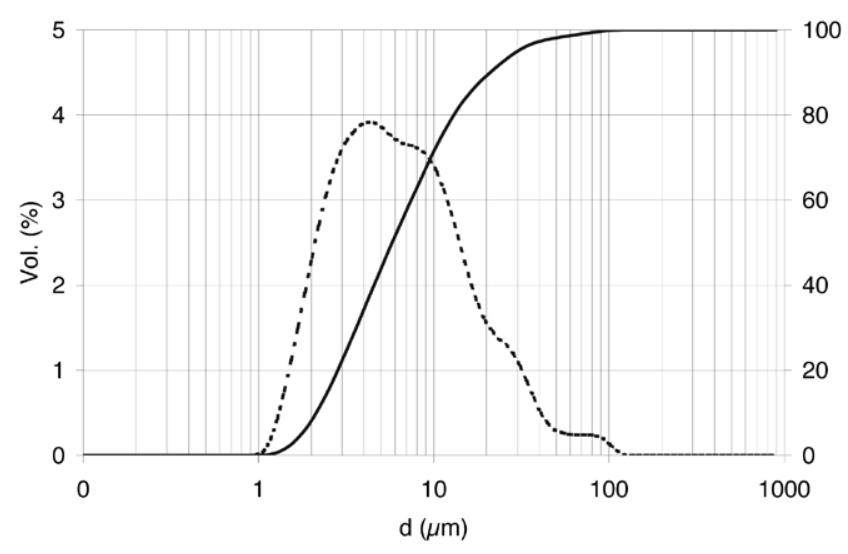

Fig. 1- Distribución de tamaños de partícula del metacaolín. 
PW2400 con tubo de Rh y provisto de un automuestreador PW2540VTC SAMPLE CHANGER. El análisis se ha realizado por dispersión de longitudes de onda, utilizando patrones de referencia que garantizan la trazabilidad de las medidas. En lo que respecta a la caracterización física se realizó el análisis granulométrico con un equipo COULTER LS 130, que mide tamaños de partículas entre 0.1 y $900 \mu \mathrm{m}$; la densidad real se midió con un picnómetro de helio y la superficie específica por el método BET con un equipo ASAP 2010 (versión 4.02) de Micromeritics. Los resultados se muestran en la tabla II y en la figura 1.

La activación alcalina se realizó utilizando tres disoluciones distintas, DN, DNW1 y DNW2, todas ellas con un porcentaje molar de $\mathrm{Na}_{2} \mathrm{O}$ próximo al $8 \%$ y porcentajes molares de $\mathrm{SiO}_{2}$ de $0.00,1.62$ y $5.40 \%$ respectivamente (Tabla III). La relación L/S (disolución activadora / metacaolín) varió en función de la disolución con el objetivo de obtener pastas de consistencia similar. En la tabla III se presentan los valores utilizados y se detallan las relaciones $\mathrm{Si} / \mathrm{Al}$ nominales, calculadas a partir de la composición del metacaolín y de las disoluciones activadoras. analizador correspondiente por energías dispersivas con un detector de estado sólido EDX OXFORD-LINK SISTEMA ISIS. La distribución de tamaños de poro se determinó con un porosímetro de mercurio de la marca Micromeritics, modelo AutoPore III. Para los espectros de ${ }^{29} \mathrm{Si}$ RMN-MAS se utilizó un equipo MSL-400 Bruker; la frecuencia de resonancia utilizada fue de $79.5 \mathrm{MHz}$, la velocidad de rotación fue $4 \mathrm{kHz}$ y como estándar se utilizó el TMS (tetrametilsilano).

\section{RESULTADOS Y DISCUSIÓN}

\subsection{Resistencia mecánica y porosidad}

En la figura 2 se presentan los resultados de la resistencia mecánica a compresión de los materiales objeto de estudio. En todos los casos se observa que, independientemente de la temperatura de curado, la matriz MK-DNW2 (mayor contenido de sílice soluble) es la que presenta mayores valores de resistencia mecánica a compresión.

En relación con el efecto de la temperatura de curado,

TABLA III. MEZCLAS DE METACAOLÍN Y SOLUCIÓN ACTIVADORA.

\begin{tabular}{|c|c|c|c|c|c|}
\hline \multirow{2}{*}{ Muestra } & \multicolumn{3}{|c|}{ Disolución activadora } & \multirow{2}{*}{ Relación L/S } & \multirow{2}{*}{ Relación teórica $\mathrm{Si} / \mathrm{Al}$} \\
\hline & Composición & $\mathrm{SiO}_{2}$ molar & Módulo $\mathrm{SiO}_{2} / \mathrm{Na}_{2} \mathrm{O}$ & & \\
\hline MK-DN & DN: $\mathrm{NaOH} 8 \mathrm{M}$ & 0.00 & --- & 1.250 & 1.16 \\
\hline MK-DNW1 & $\begin{array}{c}\text { DNW1: } 15 \% \mathrm{~W}^{(*)}+ \\
85 \% \mathrm{NaOH} 10 \mathrm{M}\end{array}$ & 1.62 & 0.19 & 1.250 & 1.27 \\
\hline MK-DNW2 & $\begin{array}{l}\text { DNW2: } 50 \% \mathrm{w}^{(*)}+ \\
50 \% \mathrm{NaOH} 13.5 \mathrm{M}\end{array}$ & 5.40 & 0.69 & 2.000 & 1.72 \\
\hline
\end{tabular}

(*) Trisilicato sódico (waterglass), porcentaje másico: $27 \% \mathrm{SiO}_{2}, 8.2 \% \mathrm{Na}_{2} \mathrm{O}, 64.8 \% \mathrm{H}_{2} \mathrm{O}$

En la preparación de probetas se utilizaron moldes metálicos de $10 \times 10 \times 60 \mathrm{~mm}$. El proceso de curado utilizado se dividió en dos etapas: un curado inicial de 3 horas a $85^{\circ} \mathrm{C}$ (Etapa 1), a continuación del cual las probetas se desmoldearon, seguido de un segundo curado (Etapa 2) de 5 horas, a diferentes temperaturas. En la tabla IV se detalla el proceso de curado realizado, que tuvo lugar, en todos los casos, con un alto nivel de humedad ambiental. Tras el curado, las probetas se mantuvieron a temperatura ambiente durante 24 horas, tiempo tras el cual se procedió a determinar su resistencia mecánica a compresión de acuerdo a la norma UNE EN 196-1: 2005 (17). El equipo utilizado para los ensayos de rotura fue una prensa Ibertest (Autotest - 200/10 - SW) con una velocidad de carga de $2.4 \mathrm{kN} / \mathrm{s}$.

El análisis mineralógico y microestructural se realizó por difracción de rayos $X$ (DRX) utilizando un equipo de la marca Philips (modelo PW-1730); mediante microscopía electrónica de barrido (SEM) utilizando un microscopio JEOL 5400 y su

TABLA IV. TIEMPO Y TEMPERATURA UTILIZADOS EN EL PROCESO DE CURADO.

\begin{tabular}{|c|c|c|}
\hline Referencia & Etapa 1 & Etapa 2 \\
\hline $\mathrm{C} 0$ & $3 \mathrm{~h}, 85^{\circ} \mathrm{C}$ & -- \\
\hline $\mathrm{C} 1$ & $3 \mathrm{~h}, 85^{\circ} \mathrm{C}$ & $5 \mathrm{~h}, 85^{\circ} \mathrm{C}$ \\
\hline $\mathrm{C} 2$ & $3 \mathrm{~h}, 85^{\circ} \mathrm{C}$ & $5 \mathrm{~h}, 150^{\circ} \mathrm{C}$ \\
\hline $\mathrm{C} 3$ & $3 \mathrm{~h}, 85^{\circ} \mathrm{C}$ & $5 \mathrm{~h}, 200^{\circ} \mathrm{C}$ \\
\hline
\end{tabular}

los datos obtenidos parecen indicar la existencia de un valor umbral a partir del cual un incremento de la temperatura de curado no se traduce en una mayor resistencia mecánica. Sin embargo, este valor umbral depende del contenido de sílice soluble en la disolución activadora. Así, para las composiciones MK-DN y MK-DNW1 (bajos contenidos de sílice soluble) este valor se corresponde con el curado $\mathrm{C} 2\left(150^{\circ} \mathrm{C}\right)$, mientras que para la composición MK-DNW2 la temperatura óptima de trabajo coincide con el curado $\mathrm{C} 1\left(85^{\circ} \mathrm{C}\right)$, con el que se llega a valores de resistencia mecánica de $19 \mathrm{MPa}$ a las 24 horas.

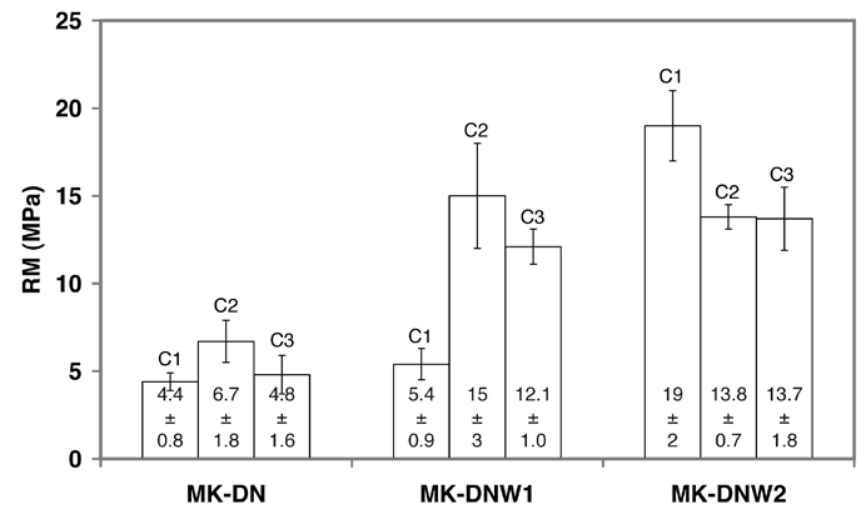

Fig. 2- Resistencia mecánica a compresión en función del proceso de curado y de la composición de la matriz. 
Respecto a la distribución de tamaños de poro, los datos de la figura 3 muestran que el material MK-DN presenta mayor volumen de intrusión total acumulado $(\approx 0.400-0.500$ $\mathrm{cm}^{3} / \mathrm{g}$ ), y, por tanto, porosidad abierta más elevada (Figura 3(a)), siendo el curado $\mathrm{C} 2$ el de menor porosidad abierta, con el que se obtienen mejores resistencias mecánicas. Esto último ocurre también con la composición MK-DNW1 (Figura 3(b)). Sin embargo, en el caso de las muestras MK-DNW2, si bien el valor de la porosidad abierta total es el mismo en todas las condiciones de curado, el diámetro medio de poros es claramente menor en el caso del curado C1 (Figura 3(c)), que se corresponde con los valores más altos de resistencia mecánica para este material. Estos resultados parecen indicar que el tipo de curado térmico, en esta composición, más que a la porosidad abierta afecta a la distribución de tamaños de poro, lo cual, a su vez, repercute notablemente en la resistencia mecánica del material.
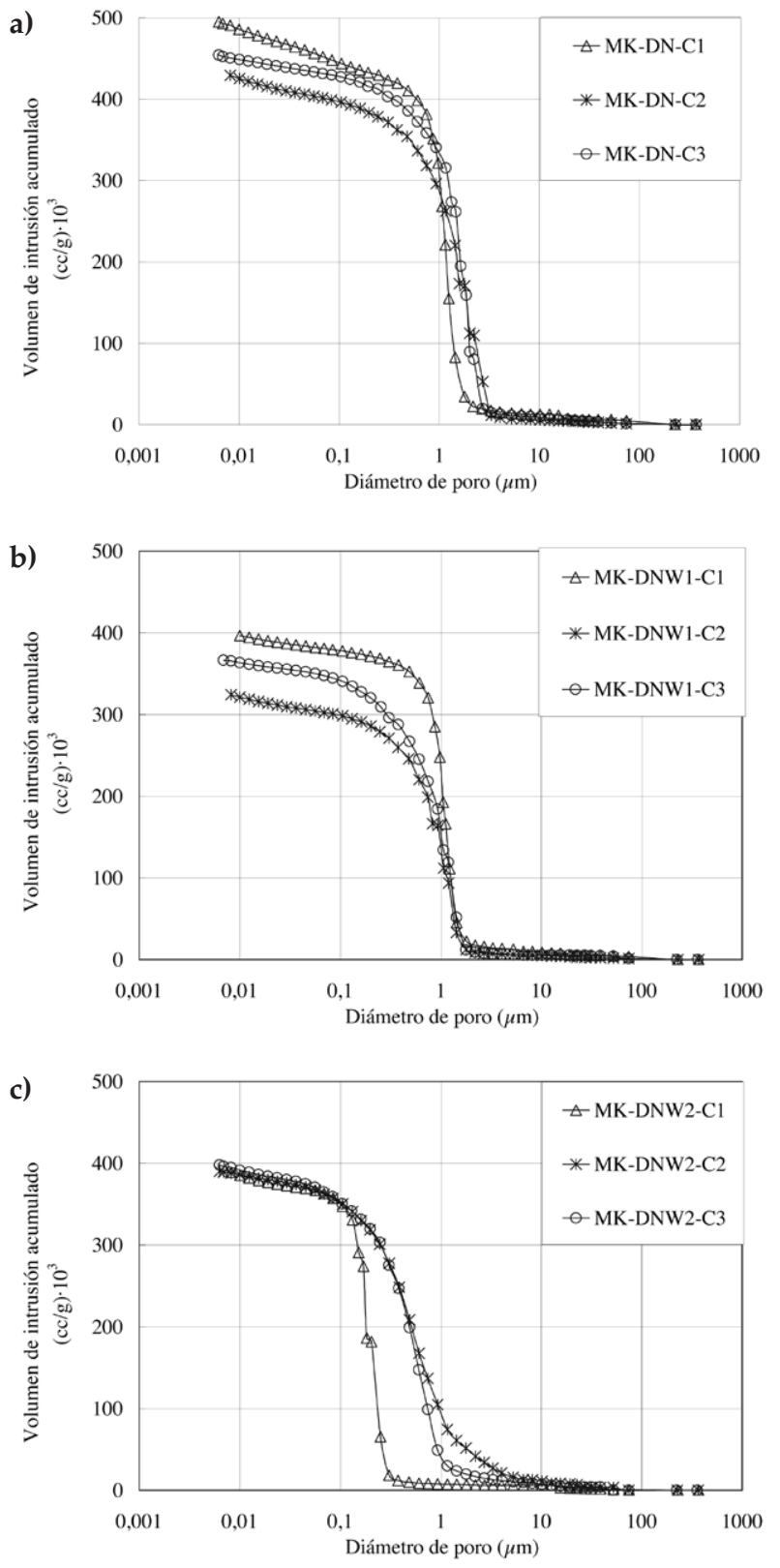

Fig. 3- Distribución de tamaños de poro en función del proceso de curado y de la composición de la matriz: a) MK-DN; b) MK-DNW1 y c) MK-DNW2.
La figura 4 muestra los valores de porosidad abierta para los tres materiales estudiados en función del proceso de curado. Comparando los resultados de las figuras 3 y 4 con los valores de resistencia a compresión de la figura 2, se observa que, si bien al disminuir la porosidad abierta aumenta la resistencia mecánica, principalmente en la matriz MK-DN, la porosidad, por si misma, no justifica los valores de resistencia obtenidos en las matrices MK-DNW1 y MK-DNW2 en los distintos procesos de curado. En estos materiales, las diferencias en la porosidad no son lo suficientemente significativas para justificar los valores de resistencia obtenidos en función de la temperatura de curado. En definitiva, para poder explicar el comportamiento mecánico observado habría que considerar no solo la porosidad o la distribución de tamaños de poro, sino también la naturaleza y composición de los productos de reacción formados, tal y como se muestra a continuación.

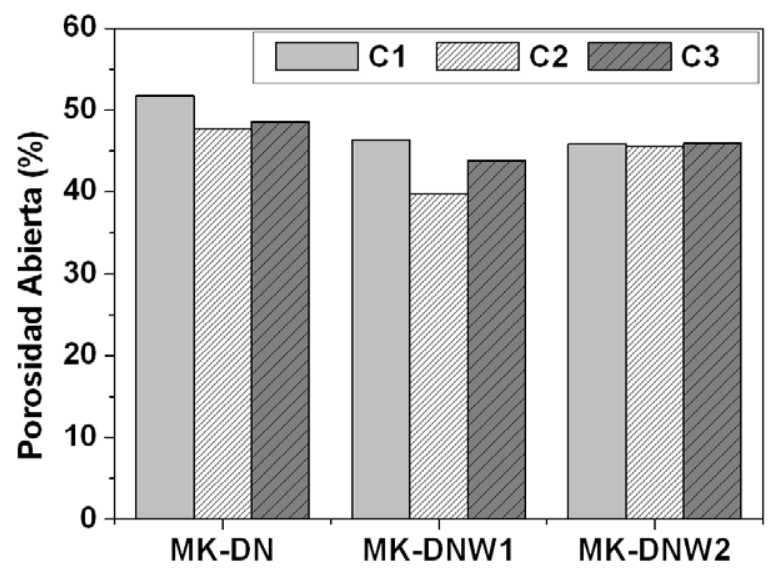

Fig. 4- Porosidad abierta en función del proceso de curado y de la composición de la matriz.

\subsection{Naturaleza y composición de los productos de reacción formados}

\subsubsection{Difracción de Rayos X (DRX)}

En la figura 5 se presentan los resultados obtenidos por difracción de rayos X. Como puede observarse, el difractograma del metacaolín de partida presenta un halo a valores de $2 \theta=20^{\circ}-30^{\circ}$ característico de los materiales amorfos, que se desplaza hacia valores de $2 \theta=25^{\circ}-35^{\circ}$ en los difractogramas de los materiales activados. Este desplazamiento indica que se ha formado, como principal producto de reacción, un polímero inorgánico alcalino, es decir, un gel N-A-S-H (8-16), de naturaleza amorfa y con propiedades cementantes.

En todos los difractogramas, bien correspondan al metacaolín o bien a los productos de activación alcalina, se observan los picos correspondientes al cuarzo (fase cristalina presente en el metacaolín), de donde se deduce que la activación alcalina, aparentemente, no ataca a esta fase en las condiciones de trabajo ensayadas.

Como fases cristalinas minoritarias se detecta la formación de zeolitas en las muestras activadas. El tipo y cantidad de zeolitas formadas depende de la matriz, mientras que la temperatura de curado solo afecta a la cantidad de zeolita formada. Así, en función de la composición del material, las zeolitas encontradas son las que se indican a continuación.

En la composición MK-DN (Figura 5(a)), que no contiene sílice soluble, es decir, que solo contiene iones $\mathrm{OH}^{-}$y $\mathrm{Na}^{+}$ 
a)

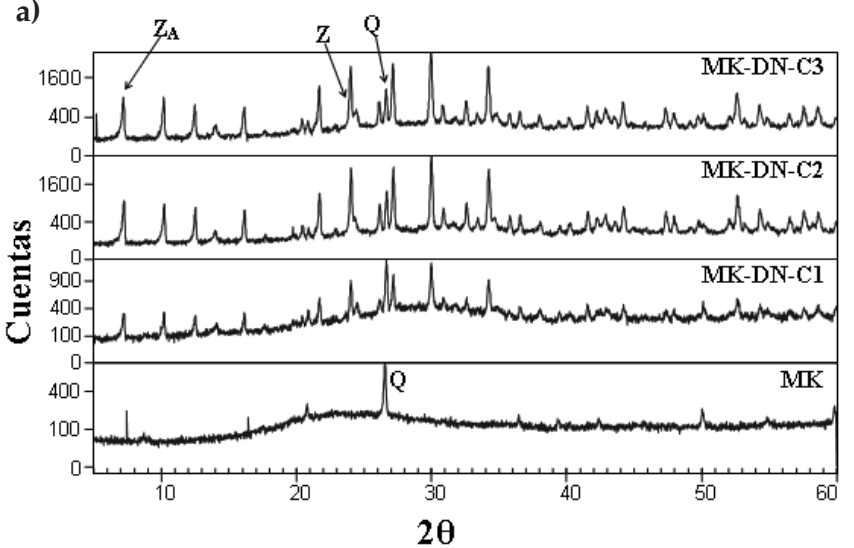

b)

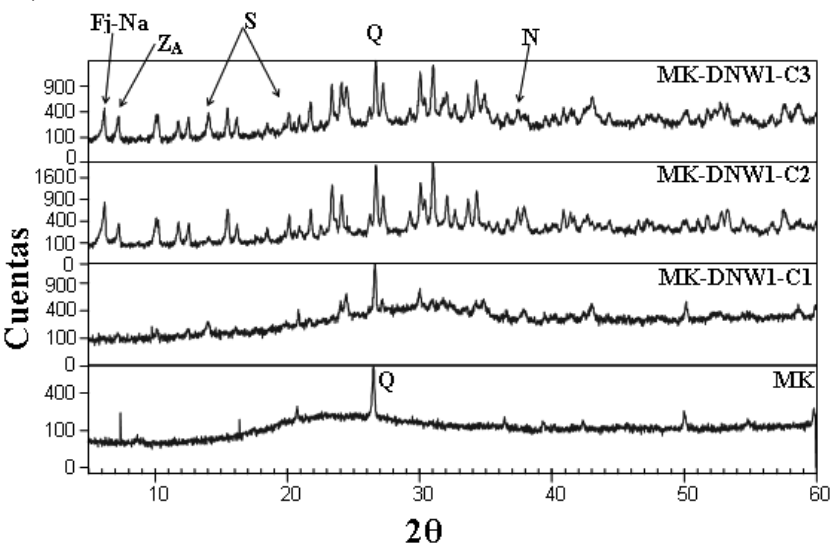

c)

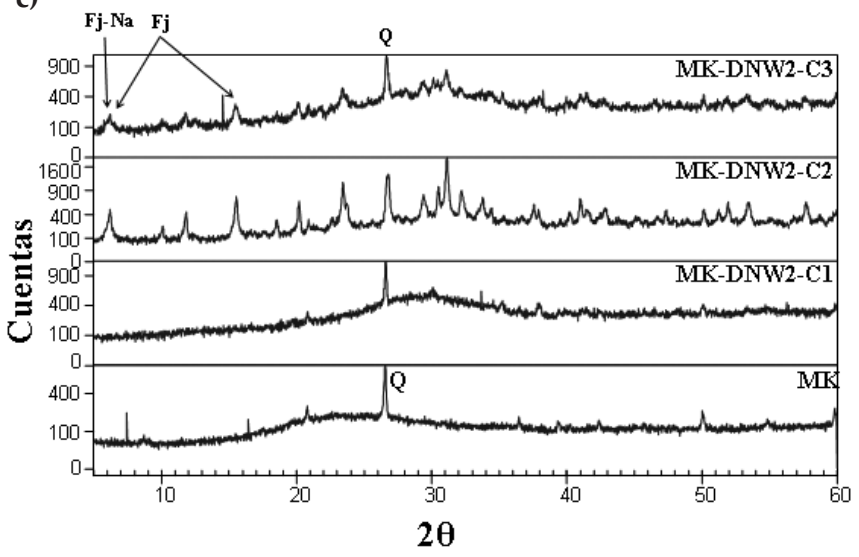

Fig. 5- Difractogramas de las diferentes matrices para los distintos procesos de curado: a) MK-DN; b) MK-DNW1; c) MK-DNW2.

(sistema de referencia), se detecta la presencia de zeolita $\mathrm{A}\left(\mathrm{Z}_{\mathrm{A}}\right)$ $\left[\mathrm{Na}_{96} \mathrm{Al}_{96} \mathrm{Si}_{96} \mathrm{O}_{384} \cdot 216 \mathrm{H}_{2} \mathrm{O}\right]$ y zeolita $(\mathrm{Z})\left[\mathrm{Na}_{6}\left(\mathrm{AlSiO}_{4}\right)_{6} \cdot 4 \mathrm{H}_{2} \mathrm{O}\right]$. Ambas zeolitas presentan una relación $\mathrm{Si} / \mathrm{Al} \approx 1$. La intensidad de sus picos es mayor en los curados C2 y C3.

En las composiciones MK-DNW1 (Figura 5(b)) se detecta la presencia de zeolita $\mathrm{A}\left(\mathrm{Z}_{\mathrm{A}}\right)$, hidroxisodalita $(\mathrm{H}-\mathrm{S})\left[\mathrm{Na}_{4} \mathrm{Al}_{3} \mathrm{Si}_{3} \mathrm{O}_{12}\right.$ $(\mathrm{OH})]$ y faujasita- $\mathrm{Na}(\mathrm{Fj}-\mathrm{Na})\left[\mathrm{Na}_{2} \mathrm{Al}_{2} \mathrm{Si}_{2.4} \mathrm{O}_{8.8} 6.7 \mathrm{H}_{2} \mathrm{O}\right]$, esta última con una relación $\mathrm{Si} / \mathrm{Al}$ mayor $(\approx 1.2)$, que se corresponde con el valor nominal, ligeramente superior, de la relación $\mathrm{Si} / \mathrm{Al}$ de este material (Tabla III). En este caso también se detecta la presencia, en bajas proporciones, de un carbonato sódico (natrita, N), asociado a procesos de carbonatación. En lo que respecta a la temperatura de curado, la mayor intensidad en los picos de las zeolitas formadas se observa, de nuevo, para los curados C2 y C3.
En la composición MK-DNW2 (Figura 5(c)) se detectan zeolitas con mayores relaciones $\mathrm{Si} / \mathrm{Al}$, como la faujasita-Na (Fj-Na) $\left[\mathrm{Na}_{2} \mathrm{Al}_{2} \mathrm{Si}_{3.3} \mathrm{O}_{10.6} \cdot 7 \mathrm{H}_{2} \mathrm{O}\right]$ y faujasita (Fj) $\left[\mathrm{Al}_{60.352} \mathrm{Si}_{139} \mathrm{O}_{3}\right.$ $\left.{ }_{71.52} \mathrm{H}_{5.984}\right]$, ambas con una elevada relación $\mathrm{Si} / \mathrm{Al}(1.7$ y 2.3 respectivamente), próximas al valor nominal de 1.72 de esta matriz. Hay que puntualizar que tanto la faujasita sódica de la composición MK-DNW1 ( $\mathrm{Si} / \mathrm{Al} \approx 1.2)$ como las de la composición MK-DNW2 ( $\mathrm{Si} / \mathrm{Al} \approx 1.7$ y / o 2.3), en ambos casos materiales activados con disoluciones que contiene sílice soluble, son de la misma familia y grupo espacial, aunque presentan reflexiones a distintos ángulos por poseer diferente composición química. Otro hecho importante a destacar es el menor grado de cristalinidad observado en las muestras con un mayor contenido de sílice soluble. Estos resultados concuerdan con los obtenidos por otros autores $(6-8,13,18$, 19) que indican que a medida que aumenta la cantidad de sílice en el medio se requiere más tiempo para que cristalicen las zeolitas.

3.2.2 Microscopía electrónica de barrido / Análisis por energías dispersivas (SEM/EDX)

Se han estudiado por microscopía electrónica las muestras correspondientes al curado $\mathrm{C} 2\left(150^{\circ} \mathrm{C}\right)$ dado que, en general, son las que presentan mayores valores de resistencia mecánica. En todos los casos, los valores de Si / Al dados se corresponden con valores medios de no menos de 10 análisis.

En la matriz MK-DN-C2, activada con la disolución de $\mathrm{NaOH} 8 \mathrm{M}$, se observa la formación, como producto mayoritario, de un gel silicoaluminoso (gel N-A-S-H), con una relación atómica $\mathrm{Si} / \mathrm{Al} \approx 1.1$ (Figura 6, punto 1 ). Como fase cristalina minoritaria se detecta la presencia de zeolita A, con una morfología de hexaedros (Figura 6, punto 2) y con una relación $\mathrm{Si} / \mathrm{Al} \approx 1$, que se corresponde con la zeolita detectada por DRX.

Cuando la disolución empleada es la DNW1 (15\% silicato soluble), se observa una matriz más densa, hecho que concuerda con los resultados obtenidos por porosimetría de $\mathrm{Hg}$ y que, en parte, justifica su mayor resistencia en comparación con la composición anterior (MK-DN). En este caso, el gel obtenido como producto mayoritario de la reacción está formado por partículas de aproximadamente $2-5 \mu \mathrm{m}$ entrelazadas y formando agregados, cuya relación atómica $\mathrm{Si} / \mathrm{Al}$ es de 1.3 (Figura 7 (a), punto 3). Como fase cristalina secundaria se observa, de nuevo, la presencia de zeolita A bien cristalizada en forma de hexaedro ( $\mathrm{Si} / \mathrm{Al} \approx 1$, Figura 7 (b) punto 4 ).

Al aumentar el contenido de silicato soluble en la disolución activadora, la matriz se densifica todavía más, como puede observarse en la figura 8 (matriz MK-DNW2-C2). Por otra parte, el gel de aluminosilicato sódico presenta una relación atómica $\mathrm{Si} / \mathrm{Al} \approx 1.7$ (punto 5 ), mayor que en el resto de muestras, lo cual concuerda con la bibliografía: la adición de silicato soluble da lugar a geles más ricos en silicio $(6-8,14$, 18-20). Todo ello justifica los mayores valores de resistencia mecánica obtenidos con esta composición. Finalmente, cabe añadir que en este material también se observa la presencia de partículas de cuarzo (punto 6) presentes en la materia prima y que no han sido atacadas en la activación, como ya se había observado anteriormente por DRX.

\subsubsection{Resonancia magnética nuclear (RMN-MAS)}

En la figura 9 se muestran los espectros de ${ }^{29} \mathrm{Si}$ RMN-MAS 


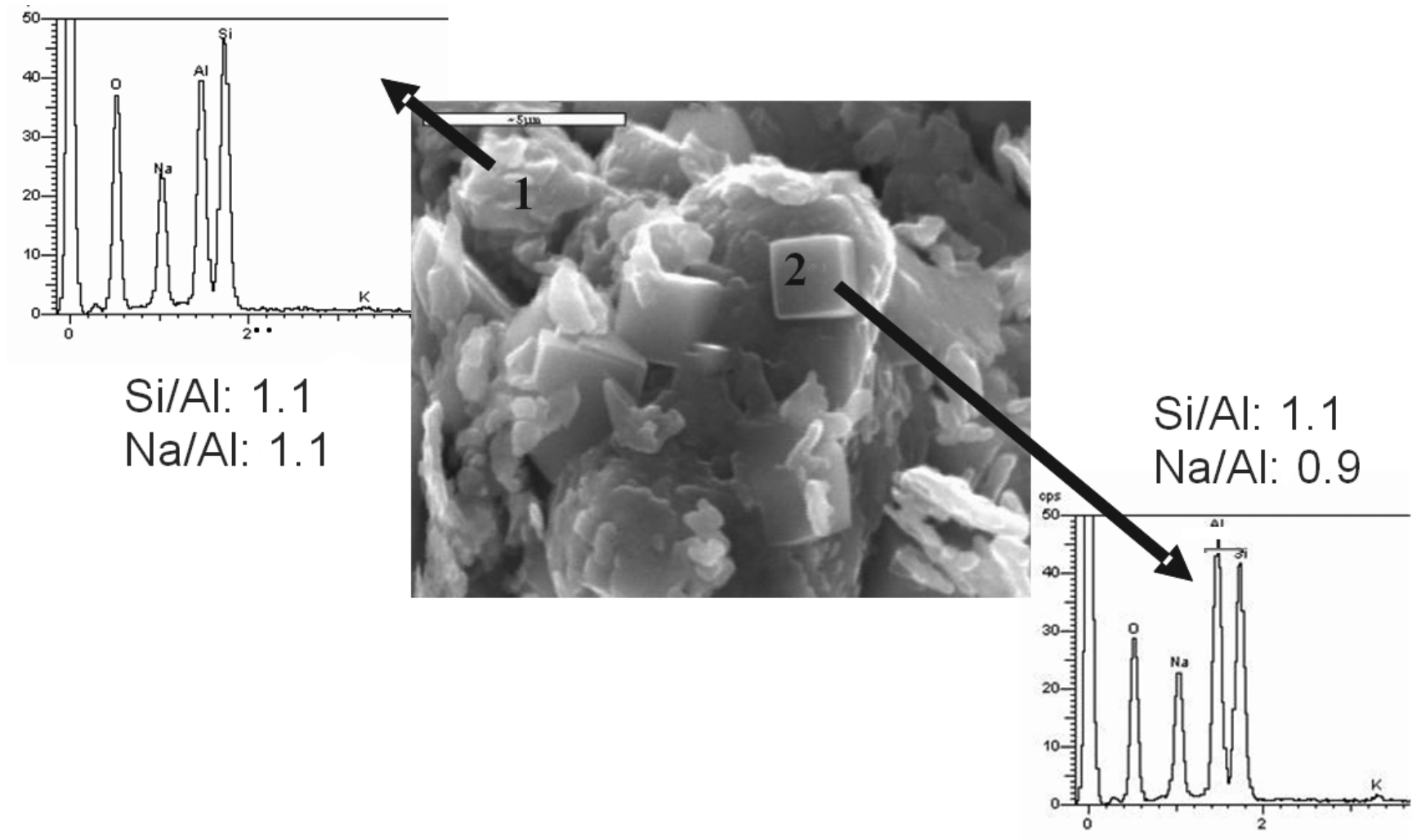

Fig. 6- Micrografía SEM y análisis EDX de la muestra MK-DN-C2.

$\mathbf{a}$

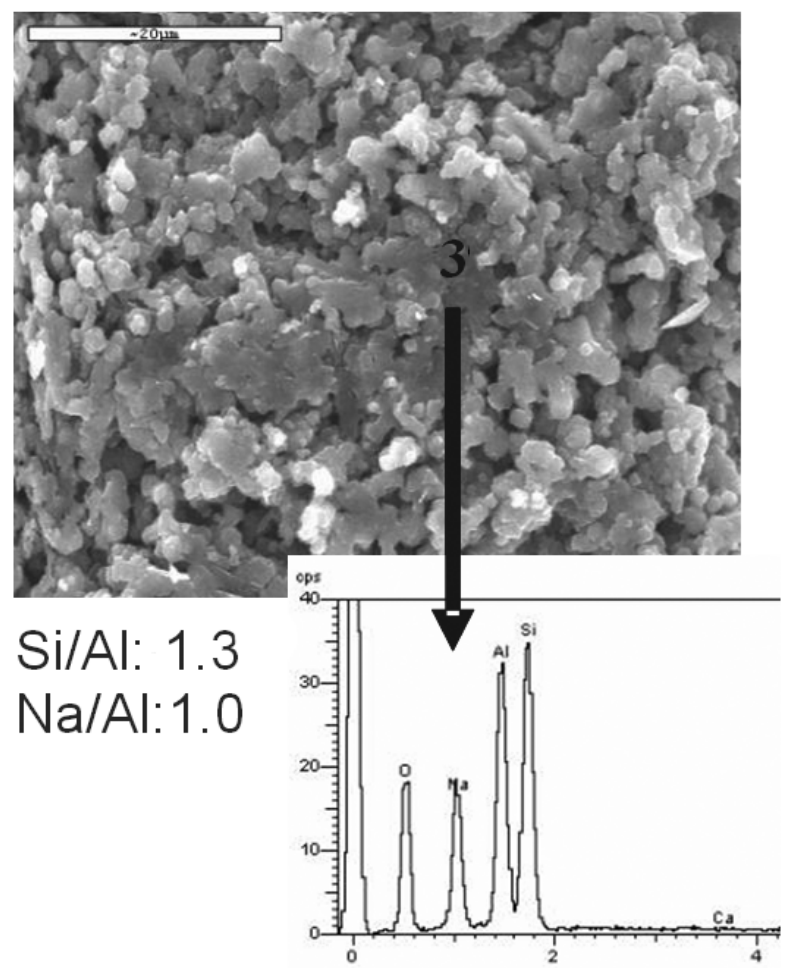

b

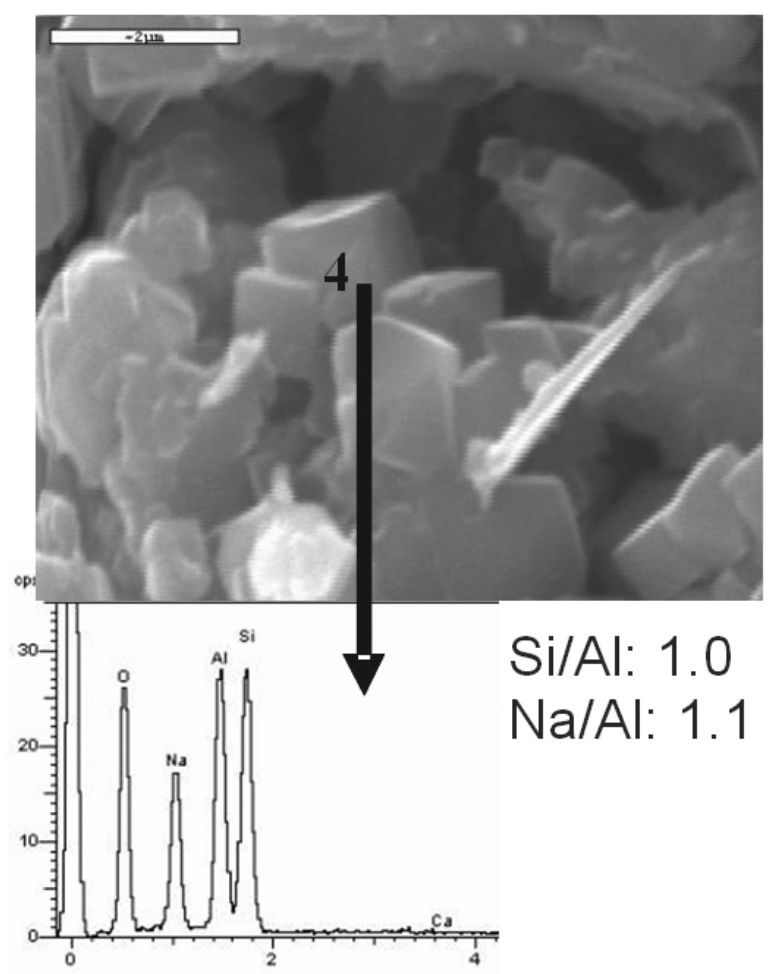

Fig. 7- Micrografía SEM y análisis EDX de la muestra MK-DNW1-C2. 


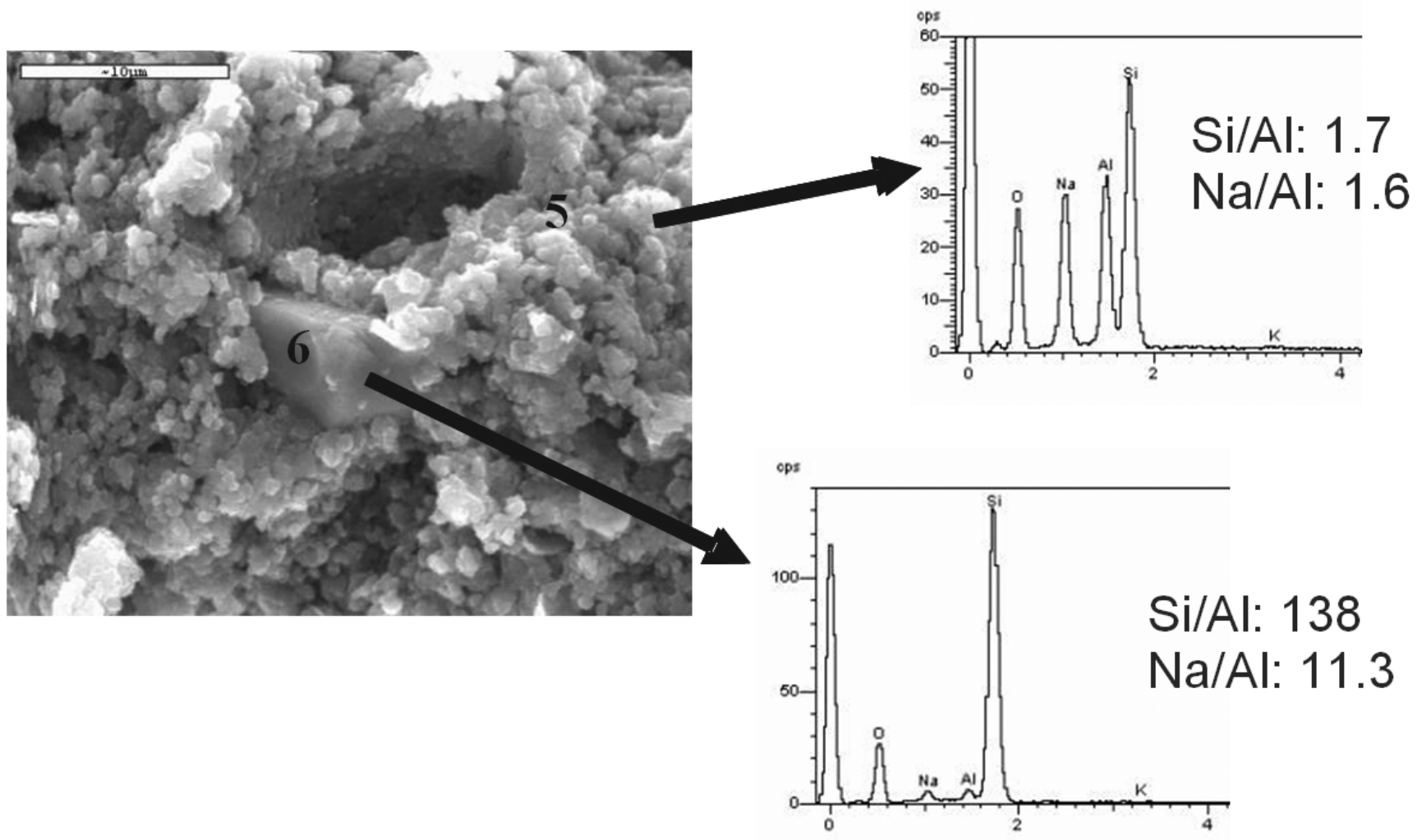

Fig. 8- Micrografía SEM y análisis EDX de la muestra MK-DNW2-C2.

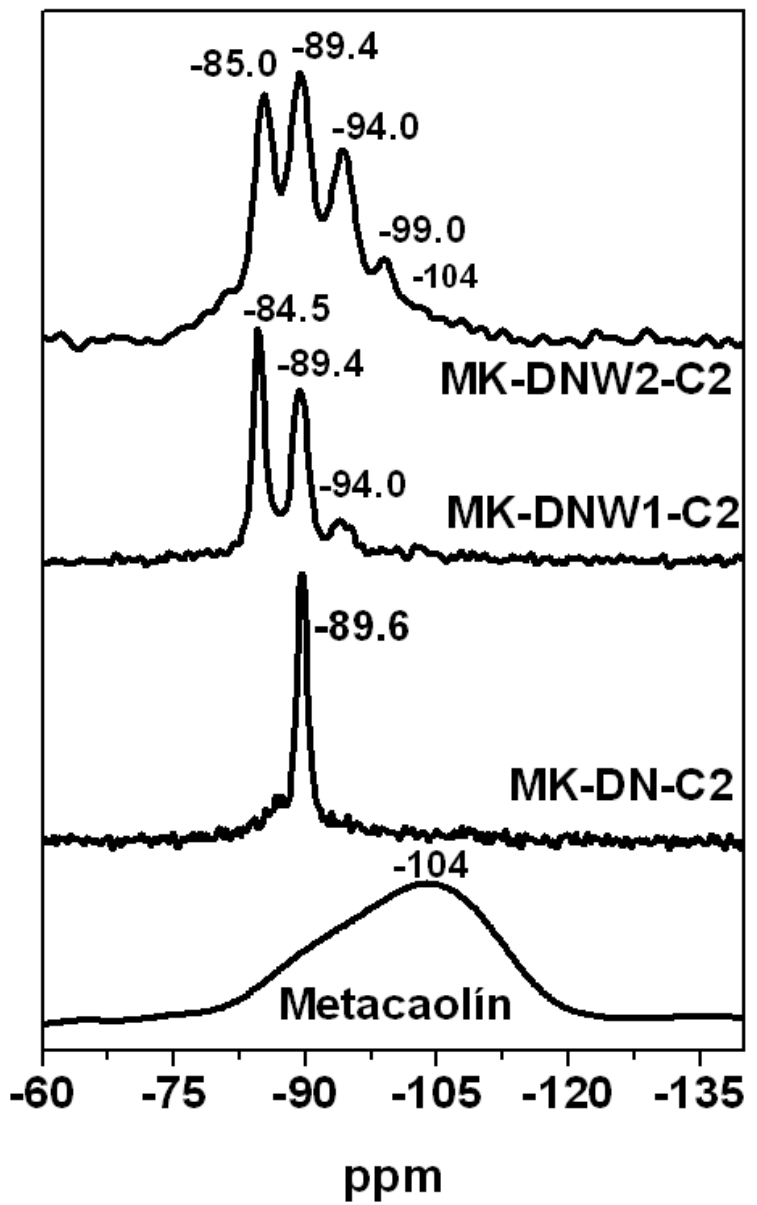

Fig. 9- Resonancia magnética nuclear de las muestras curadas en las condiciones $\mathrm{C} 2$. del metacaolín de partida y de las muestras activadas con el curado $\mathrm{C} 2\left(150^{\circ} \mathrm{C}\right)$. El metacaolín presenta una señal ancha centrada hacia -104ppm lo cual indica la heterogeneidad de la matriz en cuanto a la distribución de los átomos de Si. Por el contrario, los espectros de las muestras activadas están mejor resueltos presentando picos agudos. El espectro de la muestra MK-DN-C2 (activada con $\mathrm{NaOH}$ ) está formado por una única señal muy aguda a $-89.6 \mathrm{ppm}$, mientras que cuando en la disolución existe sílice soluble, disoluciones DNW1 y DNW2, los espectros de las muestras presentan más picos, lo que indica una mayor variedad de entornos. La asignación de los picos se realizó teniendo en cuenta los trabajos de Engelhardt (21) y de Klinowski (22), así como los datos obtenidos por DRX.

Por DRX se detecta la presencia de zeolitas tipo sodalita, zeolita A y faujasita. Según la bibliografía, la sodalita está formada por cuatro anillos que presentan en el espectro ${ }^{29} \mathrm{Si}$ RMN-MAS una única señal intensa a $-84.8 \mathrm{ppm}$ asociada, asimismo, a unidades $\mathrm{Q}^{4}(4 \mathrm{Al})$. La estructura de la zeolita A está formada por uniones de sodalita a través de anillos dobles de cuatro miembros, que presentan una única señal sobre -89ppm asociada a la presencia de unidades $\mathrm{Q}^{4}(4 \mathrm{Al})(16$, $21,22)$. Ambas zeolitas, sodalita y zeolita A, se caracterizan por presentar una relación $\mathrm{Si} / \mathrm{Al} \approx 1$, que implica que en la estructura, cada átomo de silicio está unido a cuatro átomos de aluminio y viceversa $(21,22)$. La estructura de la faujasita está formada por uniones de sodalita a través de anillos dobles de seis miembros; en función de la relación $\mathrm{Si} / \mathrm{Al}$ pueden presentar hasta cinco picos en posiciones de -84, -88, -93, -98 y $-103 \pm 1 \mathrm{ppm}$, asignados, respectivamente, a átomos de $\mathrm{Si}$ rodeados de 4, 3, 2, 1 y 0 átomos de $\mathrm{Al}(21,22)$.

Teniendo en cuenta estas consideraciones, la señal de -89.6ppm de la muestra MK-DN-C2 se asocia a entornos 
$\mathrm{Q}^{4}(4 \mathrm{Al})$ de estructuras tipo zeolita A con relaciones Si / Al $\approx 1$. El espectro de la muestra MK-DNW1-C2 presenta tres picos en posiciones de $-84.5,-89.4$ y $-94 \mathrm{ppm}$, que, considerando lo expuesto anteriormente, podrían asignarse a unidades de Si rodeadas de 4, 3, y 2 aluminios respectivamente, si bien, la elevada intensidad de la señal de -84ppm se puede interpretar considerando que en dicha señal también contribuye la sodalita, según se ha detectado en DRX. La diferente asignación realizada de la señal de -89.6/-89.4ppm en las muestras MKDN-C2 y MK-DNW1-C2 se basa en datos publicados sobre zeolitas en los que se indica que en un mismo material pueda haber átomos de silicio con entornos similares en posiciones cristalográficas no equivalentes $(21,22)$.

Finalmente, en el espectro de la muestra MK-DNW2-C2 se distinguen cinco picos a -85, -89.4, -94, -99, -104ppm. Teniendo en cuenta que, en este caso, solo se detecta la presencia de zeolitas tipo faujasita, estos picos pueden asociase a unidades tipo $\mathrm{Q}^{4}(4 \mathrm{Al}), \mathrm{Q}^{4}(3 \mathrm{Al}), \mathrm{Q}^{4}(2 \mathrm{Al}), \mathrm{Q}^{4}(1 \mathrm{Al})$ y $\mathrm{Q}^{4}(0 \mathrm{Al})$ respectivamente.

Resumiendo, los resultados de este trabajo muestran claramente que la presencia de sílice soluble en la disolución activadora mejora la resistencia mecánica del material resultante, con independencia de cual sea la temperatura de curado. La justificación de este aumento de resistencia se encuentra en la contribución de una serie de factores:

- Los datos obtenidos por SEM y RMN indican que al añadir silicato sódico soluble en la disolución activadora, aumenta la relación $\mathrm{Si}$ / Al del gel N-A-S-H formado, es decir, se forman geles más ricos en sílice. Según la bibliografía (6-8), dado que los enlaces Si-O-Si son más fuertes que los Si-O$\mathrm{Al}$, la resistencia mecánica es mayor al aumentar la relación $\mathrm{Si} / \mathrm{Al}$.

- La incorporación de una mayor cantidad de sílice soluble al medio, según los datos obtenidos por DRX y RMN, ralentiza la formación de zeolitas. Las zeolitas se consideran productos secundarios de la reacción que, en exceso, pueden debilitar la estructura $(8,13)$. De ahí que la muestra con menor cantidad de zeolitas (MK-DNW2) posea mayor resistencia mecánica.

- Los datos de porosidad confirman que al aumentar la cantidad de sílice soluble en la disolución activadora, se forma un gel más compacto. La porosidad abierta y/o el tamaño medio de poros son menores, lo cual repercute directamente en la mejora de la resistencia mecánica a compresión.

En cuanto al proceso de curado, se ha observado que, en general, la resistencia mecánica del material resultante aumenta con la temperatura de curado, hecho que concuerda con resultados obtenidos en trabajos previos en los que se demostró que el tiempo y la temperatura juegan un papel importante en el comportamiento mecánico de estos materiales $(8,15,16)$. No obstante, los resultados obtenidos muestran que existe un valor umbral a partir del cual el aumento de la temperatura de curado comienza a tener efectos negativos sobre la resistencia mecánica. El valor óptimo de la temperatura parece estar relacionado con el tipo de activador empleado, observándose que al aumentar la concentración de sílice soluble disminuye la temperatura de curado necesaria para alcanzar el valor óptimo de resistencia mecánica. Si bien las causas de este comportamiento todavía no están claras, todo parece indicar que:

- Si la temperatura se incrementa en exceso, como es el caso del curado $\mathrm{C} 3\left(200^{\circ} \mathrm{C}\right)$, la estructura se debilita, probablemente por pérdida de parte del agua de consistencia, lo que sugiere la necesidad de mantener pequeñas cantidades de agua estructural para reducir la aparición de grietas y mantener la integridad estructural del material. Por otra parte, una temperatura excesivamente elevada acelera la cinética de las reacciones, favoreciendo la cristalización de zeolitas en detrimento de la cantidad de gel N-A-S-H, de ahí que los materiales curados a $200^{\circ} \mathrm{C}$ presenten un mayor grado de zeolitización y menor resistencia mecánica.

- Estos procesos se ven acentuados cuando en el medio existe una mayor cantidad de sílice. En estos casos tienen lugar dos fenómenos contrapuestos: la mayor cantidad de sílice retrasa los procesos de cristalización y el incremento de la temperatura acelera estos procesos. Como consecuencia se forman menos zeolitas aunque con una mayor relación $\mathrm{Si} / \mathrm{Al}$ y de mayor tamaño. Ello justifica los resultados obtenidos con la composición MK-DNW2, en la que temperaturas de curado elevadas $\left(150\right.$ y $\left.200^{\circ} \mathrm{C}\right)$ tienen un efecto más perjudicial que en el resto de muestras en cuanto a la evolución de la resistencia mecánica.

\section{CONCLUSIONES}

El principal producto de reacción que se forma en los procesos de activación alcalina de metacaolín es un gel de aluminosilicato sódico (gel N-A-S-H) que posee una nanoestructura tridimensional muy similar a la que poseen las zeolitas. Este gel es el principal responsable del buen comportamiento mecánico del material. Como productos secundarios de reacción se forman zeolitas. El tipo de zeolita formada depende, principalmente, de la composición del material de partida mientras que la cantidad y grado de cristalinidad de la misma depende de la temperatura de curado.

La incorporación de una mayor cantidad de sílice soluble al medio ralentiza la formación de zeolitas y da lugar a geles más ricos en $\mathrm{Si}$. Ambos factores tienen un efecto beneficioso sobre la evolución de la resistencia mecánica del material.

La temperatura de curado acelera los procesos de reacción, en general, y más específicamente la velocidad de cristalización de las zeolitas, lo que justifica la existencia de un valor umbral de temperatura a partir del cual un aumento de la misma deja de tener efectos beneficiosos sobre la resistencia mecánica. Dicho valor umbral está directamente relacionado con la composición del material de modo que valores menores de dicha temperatura umbral se corresponden con valores superiores de sílice soluble en las muestras.

\section{AGRADECIMIENTOS}

Al Programa de Alta Especialización en Tecnologías Industriales "Nuevas técnicas y recubrimientos cerámicos de baja temperatura" (IMAETA/2004/15-IMAETB/2005/12IMAETB/2006/18) financiado por el Instituto de la Pequeña y Mediana Industria de la Generalitat Valenciana (IMPIVA) y a la Unión Europea (Fondo Social Europeo), a la Dirección General de Investigación Científica y Técnica por la financiación del proyecto BIA2004-04835 y al CSIC/Fondo Social Europeo por el contrato de investigador en prácticas REF I3P-PC2004L. 


\section{BIBLIOGRAFÍA}

1. V. Glukhovsky, The Soil Silicates, Gosstroy, Kiev, 1959. [Tesis doctoral][en ruso].

2. P. Krivenko, Alkaline cements: terminology, classification, aspects of durability, pp. 4iv046-4iv050 en $10^{\text {th }}$ International Congress on the Chemistry of Cement: Proceedings: vol 4: Performance and durability of cementitious materials. Ed. H. Justnes, ICCC, Gothenburg (Suiza) 1997.

3. J. Davidovits, Geopolymer chemistry and properties, pp. 25-48 en Geopolymers' 88: first european conference on soft metallurgy, Volume 1. Ed. J. Davidovits, J. Orlinski, Institut Géopolymère, Saint-Quentin (Francia) 1988.

4. A. Palomo, M.T. Blanco Varela, M.S. Granizo, F. Puertas, T. Vázquez, M.W. Grutzeck. «Chemical stability of cementitious materials based on metakaolin». Cem. Concr. Res. 29, 997-1004 (1999).

5. M.L. Granizo, M.T. Blanco-Varela, A. Palomo. «Influence of the starting kaolin on alkali-activated materials based on metacaolin. Study of the reaction parameters by isothermal conduction calorimetry». J. Mater. Sci. 35 [24], 6309-6315 (2000).

6. A. Fernández-Jiménez, A. Palomo. «Composition and microstructure of alkali activated fly ash binder: effect of the activator». Cem. Concr. Res. 35, 1984-1992 (2005).

7. P. Duxson, J.L. Provis, G.C. Lukey, S.W. Mallicoat, W.M. Kriven, J.S.J. van Deventer. «Understanding the relationship between geopolymer composition, microstructure and mechanical properties». Colloids Surf. A 269, 47-58 (2005).

8. P. Duxson, A. Fernández-Jiménez, J.L. Provis, G.C. Lukey, A Palomo, J.S.J. van Deventer. «Geopolymer technology: the current state of the art». J. Mater. Sci. 42, 2917-2933 (2007)

9. A. Fernández-Jiménez, I. García-Lodeiro, A. Palomo. «Durability of alkaliactivated fly ash cementitious materials». J. Mater. Sci. 42, 3055-3065 (2007).

10. T. Bakharev. «Resistance of geopolymer materials to acid attack». Cem. Concr. Res. 35, 658-670 (2005).
11. A. Fernández-Jiménez, A. Palomo, M. Criado. «Alkali activated fly ash binders. A comparative study between sodium and potassium activators». Mater. Constr. 56 [281], 51-65 (2006).

12. A. Palomo, A. Fernández-Jiménez, M. Criado. «Geopolymers: one only chemical basis, some different microstructures». Mater. Constr. 54, 77-91 (2004).

13. M. Criado, A. Fernández-Jiménez, A.G. de la Torre, A. Palomo, G. LópezOlmo, M.M. Alonso, M.A.G. Aranda. «An XRD study of the effect of the $\mathrm{SiO}_{2} / \mathrm{Na}_{2} \mathrm{O}$ ratio on the alkali activation of fly ash». Cement Concr. Res. 37, 671-679 (2007).

14. Sindhunata, J.S.J. van Deventer, G.C. Lukey, H. Xu. «Effect of curing temperature and silicate concentration on fly-ash-based geopolymerization». Ind. Eng. Chem. Res. 45, 3559-3568 (2006).

15. A. Palomo, S. Alonso, A. Fernández-Jiménez, I. Sobrados, J. Sanz. «Alkaline activation of fly ashes: NMR study of the reaction products». J. Am. Ceram. Soc. 87 [6], 1141-1145 (2004).

16. A. Fernández-Jiménez, M. Monzó, M. Vicent, A. Barba, A. Palomo A. «Alkaline activation of metakaolin-fly ash mixtures: obtained of zeoceramics and zeocements» Microporous mesoporous mater. [en prensa].

17. UNE EN 196-1:2005. Métodos de ensayo de cementos. Parte 1: Determinación de resistencias mecánicas.

18. M. Criado, A. Fernández-Jiménez, A. Palomo, I. Sobrados, J. Sanz. «Alkali activation of fly ash. Effect of the $\mathrm{SiO}_{2} / \mathrm{Na}_{2} \mathrm{O}$ ratio. Part II. ${ }^{29} \mathrm{Si} \mathrm{NMR}$ study». Microporous mesoporous Mater. [en prensa].

19. M. Criado, A. Fernández-Jiménez, A. Palomo. «Alkali activation of fly ash Effect of the $\mathrm{SiO}_{2} / \mathrm{Na}_{2} \mathrm{O}$ ratio. Part I. FTIR study». Microporous mesoporous Mater. [en prensa].

20. A. Buchwald, C. Kaps, M. Hohmann. «Alkali-activated metakaolin cement» en $7^{\text {th }}$ CANMET/ACI Conference on fly ash, silica fume, slag and natural pozzolanic in concrete, Chennai (Madras), India, 2001. [en línea] [consulta: 2007-05-29] <http://www.uni-weimar.de/Bauing/bauchemie/ Downloads/Buchwald_PosterMadras.pdf $>$

21. G. Engelhardth, D. Michel. pp. 207-230 en «High resolution solid state NMR of silicates and zeolite». Wiley, Londres (Reino Unido) 1987.

22. J. Klinowski. «Nuclear Magnetic Resonance Studies of Zeolites». Prog. Nucl. Magn. Reson. Spectrosc. 16, 237-309 (1984). 